Open Access

\title{
Wolbachia pseudogenes and low prevalence infections in tropical but not temperate Australian tephritid fruit flies: manifestations of lateral gene transfer and endosymbiont spillover?
}

\author{
Jennifer L. Morrow ${ }^{1}$, Marianne Frommer ${ }^{2}$, Jane E. Royer ${ }^{3}$, Deborah C. A. Shearman² and Markus Riegler ${ }^{{ }^{*}}$
}

\begin{abstract}
Background: Maternally inherited Wolbachia bacteria infect many insect species. They can also be transferred horizontally into uninfected host lineages. A Wolbachia spillover from an infected source population must occur prior to the establishment of heritable infections, but this spillover may be transient. In a previous study of tephritid fruit fly species of tropical Australia we detected a high incidence of identical Wolbachia strains in several species as well as Wolbachia pseudogenes. Here, we have investigated this further by analysing field specimens of 24 species collected along a 3,000 km climate gradient of eastern Australia.

Results: Wolbachia sequences were detected in individuals of nine of the 24 (37\%) species. Seven (29\%) species displayed four distinct Wolbachia strains based on characterisation of full multi locus sequencing (MLST) profiles; the strains occurred as single and double infections in a small number of individuals (2-17\%). For the two remaining species all individuals had incomplete MLST profiles and Wolbachia pseudogenes that may be indicative of lateral gene transfer into host genomes. The detection of Wolbachia was restricted to northern Australia, including in five species that only occur in the tropics. Within the more widely distributed Bactrocera tryoni and Bactrocera neohumeralis, Wolbachia also only occurred in the north, and was not linked to any particular mitochondrial haplotypes.

Conclusions: The presence of Wolbachia pseudogenes at high prevalence in two species in absence of complete MLST profiles may represent footprints of historic infections that have been lost. The detection of identical low prevalence strains in a small number of individuals of seven species may question their role as reproductive manipulator and their vertical inheritance. Instead, the findings may be indicative of transient infections that result from spillover events from a yet unknown source. These spillover events appear to be restricted to northern Australia, without proliferation in host lineages further south. Our study highlights that tropical fruit fly communities contain Wolbachia pseudogenes and may be exposed to frequent horizontal Wolbachia transfer. It also emphasises that global estimates of Wolbachia frequencies may need to consider lateral gene transfer and Wolbachia spillover that may be regionally restricted, transient and not inherited.
\end{abstract}

\footnotetext{
* Correspondence: m.riegler@westernsydney.edu.au

${ }^{1}$ Hawkesbury Institute for the Environment, Western Sydney University,

Locked Bag 1797, Penrith, NSW 2751, Australia

Full list of author information is available at the end of the article
}

(c) 2015 Morrow et al. Open Access This article is distributed under the terms of the Creative Commons Attribution 4.0 International License (http://creativecommons.org/licenses/by/4.0/), which permits unrestricted use, distribution, and reproduction in any medium, provided you give appropriate credit to the original author(s) and the source, provide a link to the Creative Commons license, and indicate if changes were made. The Creative Commons Public Domain Dedication waiver (http://creativecommons.org/publicdomain/zero/1.0/) applies to the data made available in this article, unless otherwise stated. 


\section{Background}

Wolbachia pipientis (Alphaproteobacteria) is a common endosymbiotic bacterium found in 40 to $50 \%$ of insect, arachnid and terrestrial isopod species $[1,2]$. In many host species Wolbachia is a reproductive parasite, while other host species may benefit from infections [3]. Wolbachia is mostly maternally inherited, but occasional horizontal transmission into uninfected lineages contributes to the large number of infected species [4-6]. Such switches of host lineages may result from hybridisation and introgression between closely related species [7]; sharing of ecological niches [8]; or transfer by or from parasitoids and predators [9]. The initial contact between Wolbachia of an infected with an uninfected host is the first essential step for the establishment of new Wolbachia-host associations. It must be followed by the colonisation of the host ovaries as a requirement for inheritance, as well as the induction of a reproductive manipulation or, alternatively, a positive fitness effect to promote its spread through new host populations [10, 11]. From an epidemiological perspective, this first exposure may be seen as a spillover of infectious agents from an infected source species to a new host species, and it may occur with or without its propagation in its new host species [12]. For Wolbachia, a model system for microbial symbiosis research, this initial spillover has so far not been differentiated from the more general concept of horizontal transfer itself. Most studies dealing with this phenomenon investigated horizontal transmission as the establishment of new heritable infections in new host lineages that may then express a reproductive phenotype leading to increases in infection frequencies in populations. However, not every spillover will result in the establishment of an inherited infection. Spillovers may still be detectable as non-heritable somatic and transient Wolbachia infections, or Wolbachia DNA [13] e.g. in digestive systems. It can be expected that the frequency of spillover is larger than the actual establishment of newly inherited infections, and this difference may inflate global estimates of actual infection frequencies. Another overestimation of infection frequencies (in particular in studies that rely on a single or a few marker genes) may stem from lateral transfer of Wolbachia genes into host genomes that can be seen as footprints of historic infections [14] that may occur without the presence of current infections, yet this has so far rarely been addressed in Wolbachia surveys [15].

Wolbachia spillover and with it Wolbachia horizontal transmission may be driven by ecological interactions between infected and uninfected host species, and may therefore be specific to habitat and climate. The diversity of ecological interactions is higher in tropical than temperate regions due to the increased biodiversity in the tropics [16, 17]. Within this existing conceptual framework it can be argued that Wolbachia spillover and horizontal transfer from infected to uninfected species should be more frequently observed in tropical than in temperate insect communities. However, this is not reflected in studies comparing the overall incidence of Wolbachia-infected species in arthropod communities from tropical and temperate regions that indicated parity of Wolbachia incidence across climatic zones [18-21]. On a different scale, Wolbachia prevalence within individual host species and populations was shown to vary from very low $[13,15,22]$ to fixation [23, 24] but little information is available on how this varying infection prevalence within species is distributed across extensive latitudinal clines and different climatic regions, except for the studies on the Wolbachia prevalence in Drosophila melanogaster and Drosophila simulans populations along the east coast of Australia [25-27].

We have previously investigated Wolbachia horizontal transfer between host species and potential lateral gene transfer in Australian tephritid species by analysing fruit fly communities of tropical Australia [28]. True fruit flies of the family Tephritidae encompass approximately 5,000 species worldwide, including key pests of the genera Anastrepha, Bactrocera, Ceratitis, Dacus and Rhagoletis [29]. The largest proportion of Australian tephritids belongs to the genus Bactrocera with 97 endemic species $[30,31]$. The majority originate from and are restricted to the tropical regions $[32,33]$. However, since establishment of horticultural production in Australia in the $19^{\text {th }}$ century, several fruit fly species have expanded into more temperate regions, in particular due to invasive expansion and planting of host plants [34].

Double infections of two Wolbachia strains were found in individuals of six Australian tephritid fruit fly species of the genera Bactrocera and Dacus [28]. These two Wolbachia strains were characterised with two distinct and complete multi locus sequence typing (MLST) profiles, ST-285 and ST-289. Individuals of a seventh fruit fly species also carried ST-285 as a single infection. Two more Wolbachia strains, ST-17 and ST-370, also characterised with full sets of MLST genes, were detected in one individual of these Bactrocera species, Bactrocera frauenfeldi, and both of these strains were also found in individuals of a parasitoid wasp species, Fopius arisanus (Braconidae), that is commonly found parasitising Australian fruit fly larvae (including $B$. frauenfeldi). All infected tephritid species were sampled from tropical northern Queensland where they have overlapping plant host range [32], food sources [35] and parasitoid complexes [36], perhaps providing more opportunities for Wolbachia spillover and horizontal transfer into new lineages within that environment than further south. This earlier MLST based study also revealed the presence of pseudogenised MLST genes in two species, Bactrocera peninsularis and Bactrocera perkinsi [28]. 
Here we have expanded on our previous work by surveying more fruit fly individuals (including from additional species) collected over a significantly greater area of eastern Australia, from tropical to temperate regions and over a distance of about 3,000 km, with a focus on the two widespread and economically-relevant species Bactrocera tryoni and Bactrocera neohumeralis. The main objective of our study was to assess incidence and prevalence of the four previously characterised Wolbachia strains in Australian tephritid fruit flies from geographically distant populations in order to test whether these high levels of shared Wolbachia strains found in the tropics can also be seen in other regions of Australia. We hypothesised that, due to increased species diversity and interactions, the detection of Wolbachia, including the detection of pseudogenes, may be higher in the more tropical regions of Australia. We also hypothesised that an overall low prevalence of identical Wolbachia strains in individuals of several species in the tropics without proliferation in other regions may be a manifestation of a Wolbachia spillover effect while the detection of Wolbachia pseudogenes and failed amplification of other marker genes in all specimens of a species may suggest lateral transfer of Wolbachia genes into the fly genome and the loss of infections in such species.

\section{Methods}

\section{Insect samples}

This study included field collected and laboratory specimens of fruit flies belonging to the genera Bactrocera, Dacus, Dirioxa and Ceratitis. A total of 592 flies from 24. species were field-collected in New South Wales, Queensland, the Northern Territory and the Torres Strait Islands during two sampling periods, from 1996 to 2001, and from 2012 to 2013 (Table 1 and Additional file 1). While the majority of tephritid species reside in the equatorial and tropical regions of Australia, ten of the 24 species included in this study also occur in the subtropical and temperate regions [32]. Most flies were male and collected in the summer by trapping with male attractants cue lure [37], methyl eugenol for Bactrocera visenda and zingerone for Bactrocera jarvisi [33]. Both sexes of $B$. tryoni, Bactrocera cacuminata and Dirioxa pornia were collected directly on or from infested fruit. Fly specimens were identified under a stereomicroscope using identification keys [30]. Samples were selected based on availability and to canvas a range of species and populations. Prior to 2010, Bactrocera aquilonis was recognised as a distinct species that is morphologically similar to B.tryoni but found only in the Northern Territory and Western Australia [38]. Hybridisation between these two species is considered to have occurred in the Northern Territory [32] and it has been proposed that this species be synonymised with $B$. tryoni [39]; therefore, here all flies originally classified as B. aquilonis collected from the Northern Territory have been listed as B. tryoni. The species Bactrocera papayae (sampled from the Torres Strait Islands in 1998) has also recently been synonymised with Bactrocera dorsalis [40]. A subset of the fly samples $(n=104$, mostly collected in equatorial and tropical Australia) were selected for MLST analysis and reported in Morrow et al. [28]. In addition to field-caught flies, we also screened eight females from each of the following laboratory lines: $B$. tryoni, B. neohumeralis, B. jarvisi, and B. cacuminata kept at Western Sydney University, Richmond, New South Wales; two independent B. neohumeralis lines from Cairns, Queensland, and Ceratitis capitata (Vienna 7/Mix 99) from Perth, Western Australia (Additional file 2) [41].

\section{DNA extraction, PCR, cloning and sequencing}

Genomic DNA was extracted from individual fruit fly abdomens, containing the reproductive organs, while the remainder of the specimen was stored in ethanol at $-80{ }^{\circ} \mathrm{C}$ for subsequent independent confirmation of positive results. Prior to DNA extraction, specimens were treated with $4 \%$ sodium hypochlorite (Sigma, St Louis, MO) for 5 min, then triton-X $(0.02 \%)$ and then thoroughly rinsed with Milli-Q water to reduce surface contamination. DNA from D. melanogaster line w1118, infected with Wolbachia strain $w$ MelPop [42], was used as a positive control. Insect tissue was ground in $1.5 \mathrm{~mL}$ microcentrifuge tubes with microtube pestles (Scientific Specialities Inc., Lodi, CA) and cell lysis performed overnight followed by extraction according to the GenElute Mammalian Genomic DNA Miniprep kit (Sigma) protocol. Elution of DNA from spin columns was performed with $100 \mu \mathrm{L}$ nuclease-free water. Risk of contamination was minimised by routinely replacing stock solutions, dispensing aliquots of stock reagents and using filter tips for all DNA extractions and PCRs. Although cross contamination of flies caught in the same trap or stored in the same tube of ethanol after collection has previously been shown to be unlikely [43], we have further minimised this risk by surface treatment of samples with sodium hypochlorite prior to DNA extraction (as outlined above), plus independent extraction and PCR experiments in different laboratories.

PCR-based screening of fruit fly DNA (Additional file 3) was undertaken using the Wolbachia surface protein $(w s p)$ and 16S rDNA loci. Primers for $w s p$ were $81 \mathrm{~F}$ and 691R [44] and Wsp-F and Wsp-R [45]. 16S rDNA was amplified with wspecF and wspecR [20]. Host mitochondrial cytochrome c oxidase subunit I (COI) fragments were amplified with Dick and Pat [46] as DNA quality control. Veracity of PCR results was tested by inclusion of notemplate controls. All positive amplicons were confirmed by replication and further screening with other primer sets [28]. COI and wsp amplicons were prepared for direct sequencing by treatment with a combination of $0.5 \mathrm{u}$ 
Table 1 Wolbachia prevalence in Australian fruit flies

\begin{tabular}{|c|c|c|c|c|c|c|c|}
\hline \multirow[b]{2}{*}{ Fruit Flies } & \multirow[b]{2}{*}{ Abbreviation } & \multicolumn{5}{|c|}{ Total Wolbachia prevalence } & \multirow[b]{2}{*}{ MLST ST [28] } \\
\hline & & No. individuals & No. wsp positive & No. 165 rDNA positive & $\begin{array}{l}\text { No. with } \\
\text { complete MLST }\end{array}$ & Prevalence (\%) & \\
\hline Bactrocera allwoodi & $B a$ & 1 & 0 & 0 & 0 & 0 & \\
\hline Bactrocera bryoniae & $B b$ & 51 & 4 & 3 & 4 & 7.8 & 285,289 \\
\hline Bactrocera cacuminata & $B c a$ & 21 & 0 & 0 & 0 & 0 & \\
\hline Bactrocera chorista & $B c h$ & 10 & 0 & 0 & 0 & 0 & \\
\hline Bactrocera decurtans & Bde & 6 & 1 & 1 & 1 & 16.7 & 285,289 \\
\hline Bactrocera dorsalis & Bdo & 1 & 0 & 0 & 0 & 0 & \\
\hline Bactrocera fallacis & $B f a$ & 9 & 0 & 0 & 0 & 0 & \\
\hline Bactrocera frauenfeldi & Bfr & 34 & 5 & 4 & 5 & 14.7 & $285,289,17,370$ \\
\hline Bactrocera jarvisi & $B j$ & 10 & 0 & 0 & 0 & 0 & \\
\hline Bactrocera manskii & Bma & 7 & 0 & 0 & 0 & 0 & \\
\hline Bactrocera murrayi & Bmu & 3 & 0 & 0 & 0 & 0 & \\
\hline Bactrocera neohumeralis & $B n$ & 132 & 13 & 12 & 13 & 9.8 & 285,289 \\
\hline Bactrocera quadrata & $B q$ & 9 & 0 & 0 & 0 & 0 & \\
\hline Bactrocera strigifinis & Bs & 37 & 5 & 4 & 5 & 13.5 & 285,289 \\
\hline Bactrocera tryoni & $B t$ & 190 & 4 & 4 & 4 & 2.1 & 285 \\
\hline Bactrocera visenda & $B V$ & 6 & 0 & 0 & 0 & 0 & \\
\hline Dacus absonifacies & $D a b$ & 6 & 0 & 0 & 0 & 0 & \\
\hline Dacus aequalis & Dae & 13 & 0 & 0 & 0 & 0 & \\
\hline Dacus axanus & Dax & 10 & 1 & 1 & 1 & 10 & 285,289 \\
\hline Dacus bellulus & Dbe & 3 & 0 & 0 & 0 & 0 & \\
\hline Dacus newmani & Dn & 9 & 0 & 0 & 0 & 0 & \\
\hline Dirioxa pornia & Dip & 12 & 0 & 0 & 0 & 0 & \\
\hline \multicolumn{8}{|l|}{ Pseudogenes $^{a}$} \\
\hline Bactrocera peninsularis & Bpen & 7 & 0 & 7 & 0 (fbpA only) & 0 & n.a. \\
\hline Bactrocera perkinsi & Bper & 5 & 5 & 5 & 0 (all but fts $Z$ ) & 0 & n.a. \\
\hline Total & & 592 & 38 & 41 & 33 & & \\
\hline
\end{tabular}

${ }^{a}$ Bactrocera peninsularis amplified strongly for $16 \mathrm{~S}$ rDNA and fbpA but not for wsp, and the loci are therefore possibly pseudogenes. Bactrocera perkinsi amplified both $16 \mathrm{~S}$ rDNA and wsp, however co-amplified pseudogenes for wsp, coxA, hcpA and fbpA, but lacked ftsZ [27]. Neither species was considered to harbour a true Wolbachia infection for any analyses

Exonuclease I (New England Biolabs, Ipswich, MA) and 0.25u Shrimp Alkaline Phosphatase (Promega), with incubation at $37{ }^{\circ} \mathrm{C}$ for $30 \mathrm{~min}$, then $95{ }^{\circ} \mathrm{C}$ for $5 \mathrm{~min}$, prior to sequencing by Macrogen (Seoul, Korea).

For products displaying multiple sequences through direct sequencing, wsp was PCR amplified for cloning. Amplicons were either gel-extracted using the Wizard SV Gel and PCR Clean-up System (Promega) and eluted in $25 \mu \mathrm{L}$ nuclease-free water; or used directly in the ligation reaction. Ligation was with $0.5 \mu \mathrm{L}$ pGEM-T Easy vector (Promega), 1X Rapid ligation buffer and $3 \mathrm{u}$ T4 DNA ligase (Promega). Transformation of JM109 competent cells (Promega) was performed according to the manufacturer's protocol. Colonies were smeared into a PCR tube using a sterile pipette tip and subjected to PCR using standard T7 Promoter and SP6 primers with reaction and cycling conditions as described for insect COI (Additional file 3). Positive clones, recognised by appropriately sized PCR products, were prepared for direct sequencing as described above. A minimum of three clones, but usually eight clones for each transformed ligate were selected for sequencing in both directions, using T7 Promoter and SP6 primers.

\section{DIG Southern hybridisation}

PCR amplicons were also authenticated by Southern hybridisation using DIG DNA Labelling and Detection Kit (Roche Applied Sciences, Indianapolis, IN) based on the higher sensitivity method outlined in Arthofer et al. [47]. The $w s p$ probe was generated as described in Additional file 3 , using D. melanogaster $w 1118$ DNA as template. This method was applied to all individuals, to also confirm 
the absence of wsp amplicons. Flies were classified as uninfected when repeated amplification attempts with wsp and $16 \mathrm{~S}$ rDNA were negative but COI was positive. Individuals were considered Wolbachia infected when wsp and $16 \mathrm{~S}$ rDNA primers amplified appropriately sized fragments from species for which we had established complete MLST profiles; wsp amplicons hybridised to the $w s p$ probe; and $w s p$ amplicons produced sequence homologues. The COI locus of selected infected and uninfected flies was sequenced to determine the host-mitochondrial association of Wolbachia sequences.

\section{PCR-RFLP}

Single restriction enzyme digestion was performed on wsp amplicons to test for multiple infections and to confirm the presence of no more than the two sequence types revealed via clone sequencing. The sequence differences within two of the wsp alleles found as multiple infections enabled TaqI (cuts wsp allele 661 at position 516) and SpeI (cuts wsp allele 11 at position 286) to distinguish the alleles. TaqI and SpeI (Promega) reactions were performed according to the manufacturer's protocols for $3 \mathrm{~h}$. The samples were electrophoresed on $1.2 \%$ agarose gels. Uncut, TaqI cut and SpeI uncut bands were independently excised from the gel. Samples were purified using the Wizard SV Gel and PCR Clean-up System and sequenced.

\section{Analyses of Wolbachia incidence and prevalence}

Wolbachia incidence was defined as the percentage of species with individuals that were infected with a fully characterised Wolbachia strain (MLST genes, wsp, 16S rDNA). Wolbachia prevalence was defined as the percentage of infected individuals within a species [1]. Furthermore, species for which all individuals had Wolbachia pseudogenes and incomplete marker gene sets were considered as uninfected. While we sampled an average of 24 individuals per species, sample size was limited for some species. Thus, we restricted inferences about Wolbachia incidence to species for which we had at least ten individuals from within the same collection period. This was to reduce the risk of underestimating Wolbachia incidences in species where Wolbachia occurs at low prevalence and was comparable to other recent studies of infection frequencies $[1,48]$.

For analysis of incidence, sampling locations were placed into five climate groups as defined by the Australian Bureau of Meteorology, based on their shared Köppen climate classifications: equatorial, tropical, subtropical, temperate and grasslands (Fig. 1). The latitudinal gradient was then split in half, with the midpoint set at $24^{\circ}$, south of Gladstone, Queensland. Wolbachia incidence between the northern and southern halves of the gradient was then tested through Fisher's exact test on the numbers of species with infected individuals versus species without infected individuals.

Prevalence was determined for twelve species only; species were selected based on either Wolbachia infection or availability of a minimum of 10 sampled individuals if uninfected. To incorporate the large differences in species distributions, a linear model $(\mathrm{lm})$ was fitted to the relationship of Wolbachia prevalence with individual species distribution (Additional file 4), based on the midpoint latitude of each species' geographic range as per Hancock et al. [32] and Royer and Hancock [33].

Prevalence of Wolbachia across geographic regions was only assessed for two widespread species, $B$. tryoni $(n=190)$ and B. neohumeralis $(n=132)$, as they were sampled from equatorial to temperate regions and had evidence of Wolbachia infections. The five Köppen climate groups were further divided into 13 latitudinal zones based on common sampling locations (Table 2). Infection prevalence across latitude and species was tested using multivariate generalised linear models (manyglm, negative binomial response) available in the package mvabund specifically designed for multivariate abundance data in community ecology [49] and this was performed in R 2.15 [50].

Fisher's exact test was also applied to test Wolbachia prevalence over time within five species that were polymorphic for Wolbachia infections and were collected across the two sampling periods.

\section{Phylogenetic analyses}

Phylogenetic analyses were performed for COI and wsp sequences in order to verify that mitochondrial lineages had independently acquired Wolbachia strains through horizontal transfer (and not through potential hybridisation with other species). It is also noted that phylogenetic analyses were not undertaken to infer species relationships, as single gene approaches are insufficient for such questions. DNA sequences were trimmed and edited in Sequencher 4.0 (GeneCodes Corporation) and then analysed in MEGA 5.05 [51]. COI and wsp genes were independently aligned (MUSCLE algorithm). Pairwise distance matrices were calculated for $\mathrm{COI}$ using number of differences and p-distance models. Substitution models were selected using Find Best DNA Model (ML), which calculated the lowest Bayesian Information Criterion score $(\mathrm{GTR}+\mathrm{G}$ for $w s p$; TN93 + G for COI). Bayesian Inference phylogenies were produced by MrBayes 3.2 [52] running $10^{7}$ generations with a sample frequency of 100 . The first $25 \%$ of trees were discarded, and a $50 \%$ majority rule consensus tree returned.

\section{Results}

Wolbachia-specific primers for wsp and 16S rDNA were used to screen 592 field-collected Australian fruit flies 


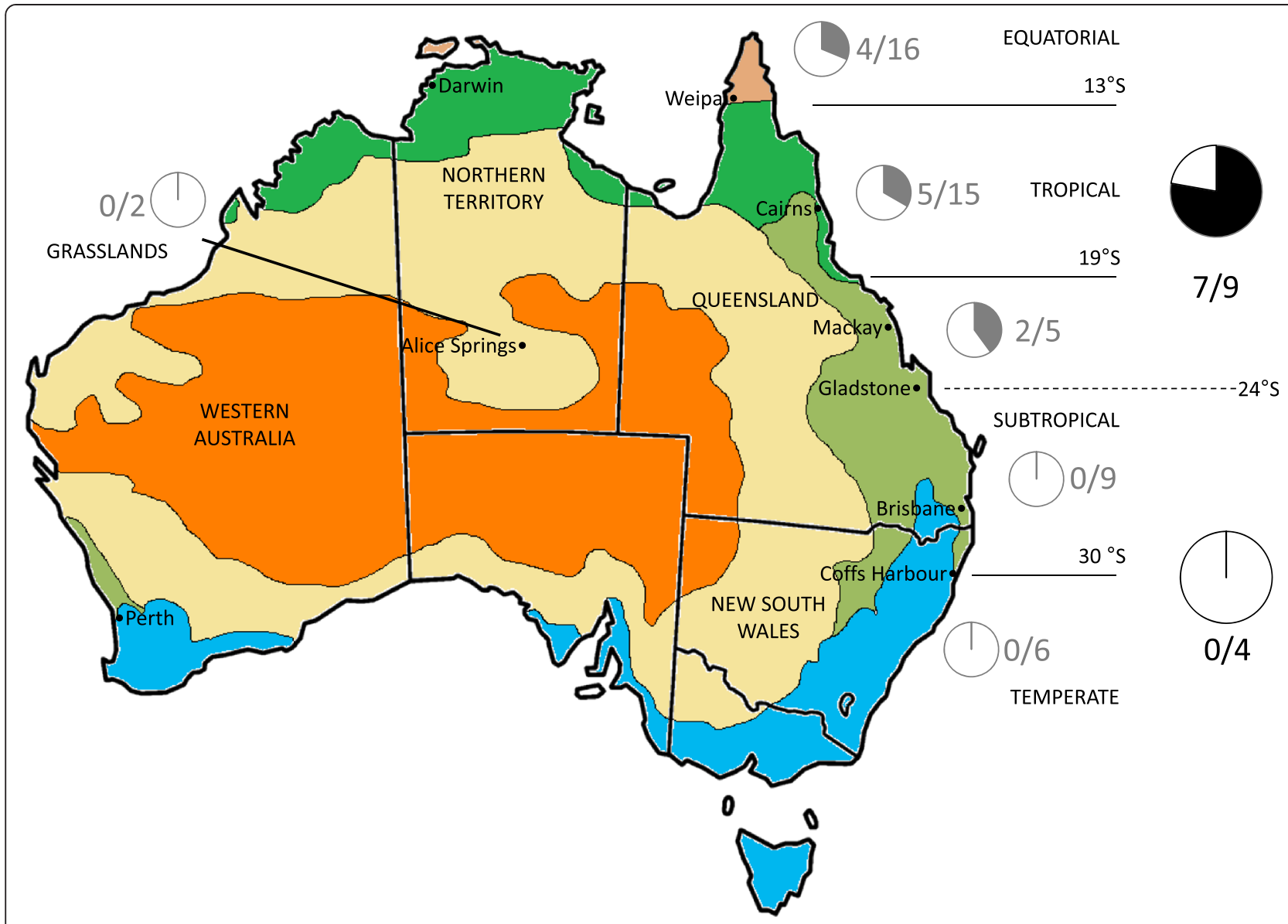

Fig. 1 Incidence of Wolbachia infection in tephritid species. The Köppen map of Australia represents the climate groups and latitudes as approximate climate divisions along the east coast of Australia. Wolbachia incidence is presented as filled sections of pie graphs, with the number of infected species per total number of tested species within each of the six regions. Grey pie graphs represent Wolbachia incidence in all studied species of individual regions. Black pie graphs represent Wolbachia incidence in species for which at least ten individuals were available north or south of $24^{\circ}$ latitude (set as midpoint for the analysis). Wolbachia incidence was higher in the northern half than the southern half of the latitudinal gradient (Fisher's exact test; $p<0.05$ )

representing 24 species of Bactrocera, Dacus and Dirioxa. Overall, individuals of eight species were positive for both wsp and $16 \mathrm{~S}$ rDNA, while all individuals of one species, B. peninsularis, were positive for $16 \mathrm{~S}$ rDNA without amplification of wsp (Table 1). All individuals from the independently established laboratory lines were negative for Wolbachia (Additional file 2). Initial screening using wsp primers $81 \mathrm{~F}$ and 691R [44] appeared to produce false positives for some flies. Thus, primers Wsp-F and Wsp-R [45] were chosen, as their amplicons were more consistent and only occasionally produced spurious bands. Southern hybridisation with wsp was applied to all 592 samples, including those that initially appeared to be negative for Wolbachia; this confirmed specificity as well as improved detection sensitivity. In this way, four additional individuals, one each from four species (B. bryoniae, B. frauenfeldi, B. neohumeralis and $B$. strigifinis) with very faint wsp PCR amplification and undetectable 16S rDNA fragments using standard PCR were confirmed to carry wsp DNA by Southern hybridisation, while other individuals in these species were positive for both loci after standard PCR. We considered these four individuals infected with low titre Wolbachia because we had previously characterised complete MLST profiles in other individuals of these host species. Furthermore, control Southern hybridisation to samples with high titre infections demonstrated that the DIGlabelled wsp probe did not bind to primer-dimers or spurious wsp products, but hybridised to $w s p$ amplicons that were verified by sequencing.

\section{Sequence analysis of wsp and $16 \mathrm{~S}$ rDNA}

Thirty-eight flies amplified at the wsp locus and 34 of these also successfully amplified at the 16S rDNA locus. Sequence analysis identified five different $w s p$ alleles of supergroup A. Two wsp alleles (661 and 11) were found 
Table 2 Wolbachia prevalence across climate zones in two widely-distributed Australian fruit fly species

\begin{tabular}{|c|c|c|c|c|c|c|c|c|c|c|c|c|c|c|c|c|c|}
\hline & & $\begin{array}{l}\text { Total } \\
\text { Wolb } \\
\text { preva }\end{array}$ & $\begin{array}{l}\text { I } \\
\text { pachia } \\
\text { alence }\end{array}$ & & Wolbachic & ia prevalence $\mathrm{p}$ & per climate & e zone (in & fected/total) & & & & & & & & \\
\hline & \multirow{3}{*}{ Abbreviation } & \multirow{3}{*}{ No. } & \multirow{3}{*}{ +ve } & \multirow{3}{*}{$\%$} & \multicolumn{2}{|l|}{ Equatorial } & \multicolumn{3}{|l|}{ Tropical } & \multicolumn{5}{|c|}{ Subtropical } & \multicolumn{2}{|l|}{ Temperate } & \multirow{2}{*}{$\begin{array}{l}\text { Grasslands } \\
\text { Alice Springs }\end{array}$} \\
\hline \multirow[t]{2}{*}{ Fruit Flies } & & & & & $\begin{array}{l}\text { Torres } \\
\text { Strait }\end{array}$ & Weipa/Coen & Darwin & Cairns & Townsville & Mackay & Gladstone & Bundaberg & Brisbane & Lismore & Coffs Harbour & Richmond & \\
\hline & & & & & $10-11^{\circ} \mathrm{S}$ & $12-13^{\circ} \mathrm{S}$ & $10-12^{\circ} \mathrm{S}$ & $16-17^{\circ} \mathrm{S}$ & $18-19^{\circ} \mathrm{S}$ & $20-21^{\circ} \mathrm{S}$ & $22-23^{\circ} \mathrm{S}$ & $24-25^{\circ} \mathrm{S}$ & $26-27^{\circ} \mathrm{S}$ & $28-29^{\circ} \mathrm{S}$ & $30-31^{\circ} \mathrm{S}$ & $32-34^{\circ} \mathrm{S}$ & $30-31^{\circ} \mathrm{S}$ \\
\hline B. neohumeralis & $B n$ & 132 & 13 & 9.8 & $0 / 11$ & n.d & & $9 / 37$ & $0 / 12$ & $3 / 12$ & $1 / 10$ & 0/10 & $0 / 28$ & $0 / 10$ & $0 / 2$ & & \\
\hline B. tryoni & $B t$ & 190 & 4 & 2.1 & n.d & $0 / 13$ & $0 / 6$ & $2 / 40$ & 0/10 & $2 / 12$ & $0 / 12$ & 0/10 & $0 / 37$ & n.d & $0 / 11$ & 0/19 & $0 / 20$ \\
\hline Total & & 322 & 17 & & 0/11 & 0/13 & $0 / 6$ & $11 / 77$ & $0 / 22$ & $5 / 24$ & $1 / 22$ & $0 / 20$ & $0 / 65$ & 0/10 & 0/13 & 0/19 & $0 / 20$ \\
\hline
\end{tabular}

The latitudinal gradient of eastern Australia was divided into regions and represented by a major town or city. Darwin and Alice Springs are included as separate areas because they are not along the east coast of Australia. Regions are classified into climate groups according to the Köppen classification (Australian Bureau of Meteorology). Bactrocera neohumeralis does not occur in Darwin, Richmond or Alice Springs [32], whereas non-determined (n.d.) regions were not sampled 
to co-occur in individuals of six species (B. bryoniae, $B$. decurtans, B. frauenfeldi, B. neohumeralis, B. strigifinis and D. axanus); beyond this, wsp 661 occurred as a single sequence in one B. frauenfeldi, four B.tryoni and three $B$. neohumeralis individuals; and wsp 11 in one $B$. neohumeralis individual (Table 3, Fig. 2). Wolbachia MLST characterisation linked wsp 661 and wsp 11 with strains ST-285 and ST-289 respectively, demonstrating a high degree of sharing of Wolbachia strains among six tephritid species [28]. PCR-RFLP and sequencing confirmed the absence of any other detectable wsp variants in these individuals.

Cloning of $w s p$ from one B. frauenfeldi individual (ID136) revealed two alleles unlike those found in the other $B$. frauenfeldi individuals: one sequence identical to wsp 16 of Drosophila simulans strain $w \mathrm{Ri}$ [53]; the other [GenBank: KC693012] with high similarity to two wsp sequences detected in B. dorsalis from China [22]. Furthermore, the wsp and MLST alleles were shared with the Wolbachia infections found in the parasitoid F. arisanus [28].

All five B. perkinsi individuals produced $w s p$ and $16 \mathrm{~S}$ rDNA amplicons. Cloning and sequencing of the wsp fragments revealed a novel allele, wsp 662, as well as multiple clones of a wsp sequence with a single base insertion. This insertion disrupts the open reading frame by incorporating stop codons, and may represent a $\mathrm{Wol}$ bachia pseudogene transferred to the host genome of this species. Amplification of the fts $Z$ locus in all individuals of $B$. perkinsi have previously failed during the MLST characterisation of Wolbachia in this species while all other loci were positive with A group like sequences [28]. We interpreted this as presence of multiple pseudogenes and absence of true Wolbachia infections in the analysed specimens of this species.

For $B$. peninsularis, the 16S rDNA fragment was consistently amplified from all seven individuals, whereas the $w s p$ locus failed to amplify. Direct sequencing of the 16S rDNA amplicon showed multiple peaks in the sequence chromatogram indicating two distinct sequences: one full length B group 16S rDNA sequence, and one sequence with a 31 bp deletion at positions 182-213, indicative of a potential pseudogene. The sequence of the $B$. peninsularis full-length $16 \mathrm{~S}$ rDNA fragment was deposited in GenBank, accession number KC775793. Failure to detect a wsp sequence in any of these individuals, and prior failure to detect the five MLST markers except $f b p A$ (new allele 196) [28], suggested that this sequence may not represent a genuine Wolbachia infection; consequently this species was classified as uninfected. As a control for the 16S rDNA PCR assays, one $B$. neohumeralis sample, ID248, was chosen for $16 \mathrm{~S}$ rDNA sequencing to confirm homology to other $\mathrm{Wol}$ bachia 16S rDNA sequences in GenBank (KC775794. and KC775795).

Table 3 wsp allele numbers and GenBank accession numbers of analysed individuals

\begin{tabular}{|c|c|c|c|c|c|}
\hline Species (double/single infection) & Individual ID No. & 16SrRNA & wspt & wsp GenBank accession no. & MLST ST [28] \\
\hline \multirow[t]{2}{*}{ B. bryoniae (d) } & $157,536,545$ & yes & 11 & KC668327 & 289 \\
\hline & & & 661 & KC668326 & 285 \\
\hline \multirow[t]{2}{*}{ B. decurtans (d) } & 85 & yes & 11 & KC668325 & 289 \\
\hline & & & 661 & KC668324 & 285 \\
\hline \multirow[t]{2}{*}{ B. frauenfeldi (d) } & 136 & yes & 16 & KC668321 & 17 \\
\hline & & & a & KC693012 & 370 \\
\hline \multirow[t]{2}{*}{ B. frauenfeldi (d) } & 485,492 & yes & 11 & & 289 \\
\hline & & & 661 & & 285 \\
\hline B. frauenfeldi (s) & 490 & yes & 661 & & 285 \\
\hline \multirow[t]{2}{*}{ B. neohumeralis (d) } & $35,109,160,221,238,248,342,345,355$ & yes & 11 & KC668323 & 289 \\
\hline & & & 661 & KC668320 & 285 \\
\hline B. neohumeralis (s) & 244 & yes & 11 & KC668323 & 289 \\
\hline B. neohumeralis (s) & $240,243,346$ & yes & 661 & KC668320 & 285 \\
\hline \multirow[t]{2}{*}{ B. strigifinis (d) } & $81,269,503,504$ & yes & 11 & KC668329 & 289 \\
\hline & & & 661 & KC668328 & 285 \\
\hline B. tryoni (s) & 275,276 & yes & 661 & KC668332 & 285 \\
\hline \multirow[t]{2}{*}{ D. axanus (d) } & 88 & yes & 11 & KC668331 & 289 \\
\hline & & & 661 & KC668330 & 285 \\
\hline B. perkinsi (pseudogene) & 74 & yes & 662 & KC668319 & n.a. \\
\hline
\end{tabular}




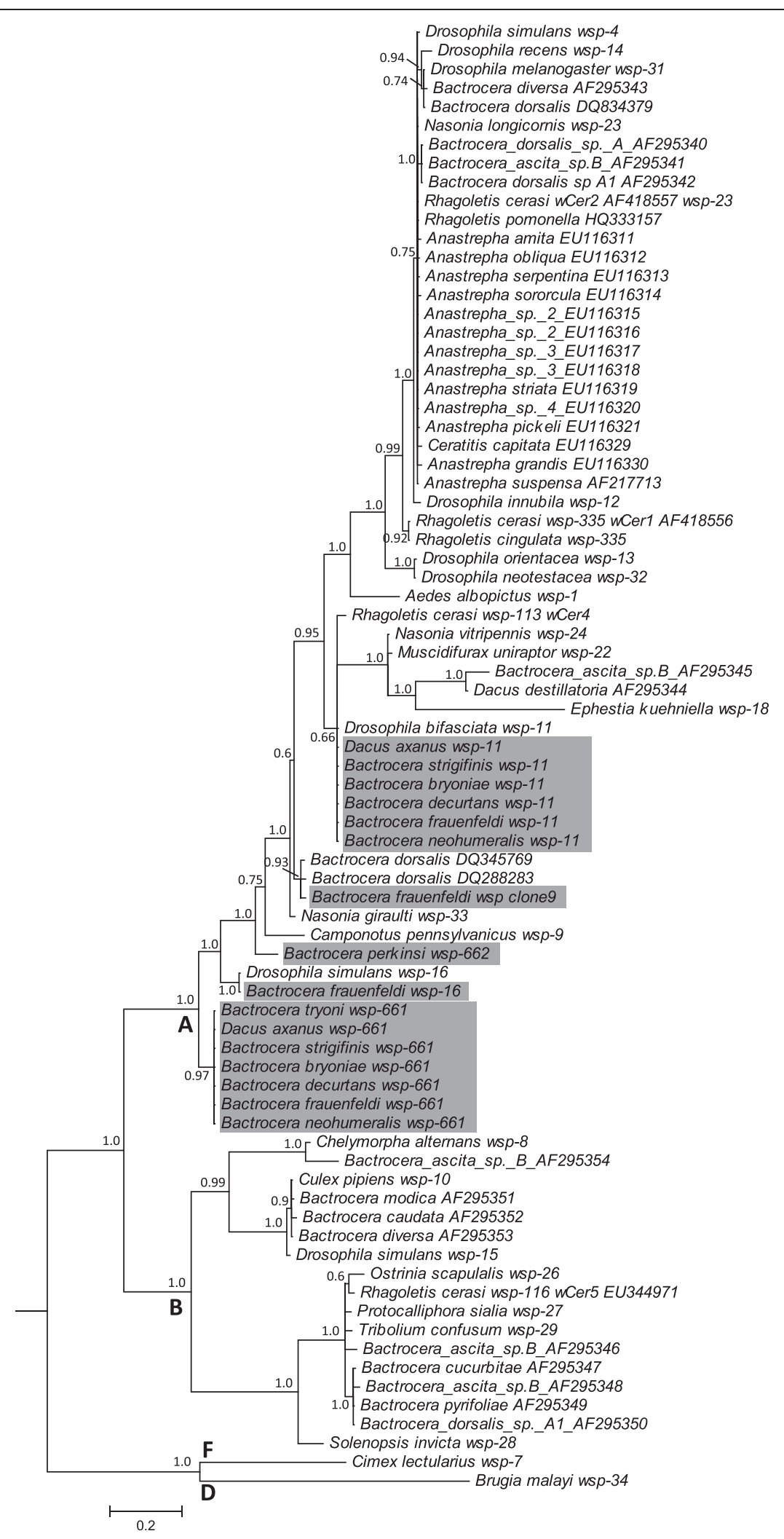

Fig. 2 (See legend on next page.) 
(See figure on previous page.)

Fig. 2 Bayesian inference tree of wsp sequences from Wolbachia within tephritid fruit flies and other invertebrates. Highlighted sequences are from this study (details in Table 3). All other sequences were retrieved from GenBank or the Wolbachia wsp database (accession number or wsp allele number following host species name). Supergroup classification of wsp sequences is marked at the nodes. Posterior probabilities $>0.50$ are marked at the nodes; tree was rooted by supergroup D and F strains. Scale bar represents the number of nucleotide substitutions per site

\section{Analysis of Wolbachia incidence and prevalence}

Geographically as well as irrespective of host species and Wolbachia strains, Wolbachia was detected in five of 131 samples from equatorial Queensland (3.8\%), 22 of 195 (11.3\%) samples from tropical Queensland and the Northern Territory, and 6 of 179 (3.4 \%) samples from subtropical Queensland. However, none of the 60 samples from temperate New South Wales and none of the 27 samples from central Australia had detectable Wolbachia (Additional file 1). The analysis of Wolbachia incidence within species revealed that it was restricted to the northern half of Australia (Fig. 1); analysis of species for which at least ten individuals per species were available for each half revealed Wolbachia incidence in at least one specimen for a number of these species in the northern half of the gradient while none was infected in the southern half. Therefore, incidence of Wolbachia was higher in the northern half of the gradient, with Wolbachia incidence in seven out of nine species for which a minimum of ten individuals were tested; all four species from the southern half for which a minimum of ten individuals per species were available in that region, were negative (Fisher's exact test; $p=0.021$ ). A linear model was applied in order to appropriately represent both widespread and more tropically restricted species and this detected a subtle relationship between the prevalence of Wolbachia and the midpoint latitude of twelve individual species $\left(R^{2}=0.86\right.$, $\mathrm{F}_{1,10}=71.43 ; p<0.001$; Additional file 4).

For the seven species that had individuals with a full Wolbachia MLST profile plus wsp, the prevalence ranged from $2.1 \%(4 / 190)$ to $16.7 \%$ (1/6; Table 1). Multivariate analysis of infection prevalence in the only two widespread species that are endemic from equatorial to temperate climes, B. neohumeralis and B. tryoni (Table 2), detected a significant interaction of Wolbachia prevalence by latitude (Dev $=38.49, p<0.05$; Table 4$)$ with Wolbachia being more common in the northern regions of east coast Australia. Univariate analysis with adjusted p-values (p.uni $=$ adjusted) to account for family-wise error across both species did not detect a significant interaction $(p>0.05)$, demonstrating that the detectable effect on a single species is weak, but the pattern is strengthened when analysing both species together.

Furthermore, temporal effects on Wolbachia prevalence within B. bryoniae, B. frauenfeldi, B. neohumeralis, B. strigifinis and B.tryoni were tested for equatorial and tropical samples by using a Fisher's exact test $(\alpha=0.05)$ over the sampling years. No significant temporal change within the tested regions was detected, except for B. tryoni $(p<0.05)$, but this represented neither an overall increase nor decrease in prevalence over time (Additional file 5).

\section{Analysis of mitochondrial DNA}

Most tephritid species produced clear COI sequences. Bactrocera jarvisi, B. murrayi, B. dorsalis and B. perkinsi individuals produced ambiguous sequences, indicative of potential nuclear mitochondrial (numt) DNA in these species. The latter sequences were not included in phylogenetic analysis. Instead, B. jarvisi and B. dorsalis sequences were retrieved from GenBank. Bayesian analysis of 81 sequences (52 sequences from this study) over $571 \mathrm{bp}$ returned a well-supported consensus tree (Fig. 3).

Mitochondrial haplotypes of morphologically identified individuals of different species harbouring identical wsp alleles were different, except for the two sibling species B. neohumeralis and B. tryoni; both had individuals with identical Wolbachia and mitochondrial sequences but without any linkage between Wolbachia infection and haplotypes. COI distance measures of B. neohumeralis and B. tryoni (analysed as one species complex) showed little difference within infected $(1.22 \%)$ and uninfected individuals (1.36\%), and between infected and uninfected individuals (1.31 \%; Additional file 6; Fig. 3).

\section{Discussion}

We surveyed 24 Australian tephritid fruit fly species throughout their natural range, and detected Wolbachia sequences in individuals of nine species. In two species, all individuals had pseudogenes and incomplete sets of marker genes - indicative of potential lateral gene transfer to the host genome without the presence of an existing infection. Both species are restricted to the tropics. The remaining seven fruit fly species had both $16 \mathrm{~S}$ rDNA and wsp sequences characteristic for four Wolbachia strains for which complete MLST profiles had previously been established [28]. The four Wolbachia strains occurred as paired or single infections in more than one host species, indicative of horizontal Wolbachia transmission events into these species. Overall the incidence of Wolbachia strains (mostly two strains, ST285 and ST-289) was restricted to tropical regions, and the prevalence of the four Wolbachia strains was very low in infected species (2-17\%). In five infected species (with enough samples available to test for a time effect), Wolbachia prevalence appeared to not have changed 
Table 4 Analyses of Wolbachia prevalence at different latitudes of B. neohumeralis and B. tryoni

\begin{tabular}{|c|c|c|c|c|}
\hline \multirow{2}{*}{$\frac{\text { Analysis of Deviance }}{\text { Multivariate test: }}$} & \multicolumn{4}{|c|}{ Model: manyglm(formula = WolbPrevalence $\sim$ latitude *status, family = "negative.binomial") } \\
\hline & Residual Df & Df & Deviance & $\operatorname{Pr}(>\mathrm{Dev})$ \\
\hline intercept & 39 & & & \\
\hline latitude & 30 & 9 & 16.47 & 0.481 \\
\hline status & 29 & 1 & 123.06 & $0.001^{* * *}$ \\
\hline latitude:status & 20 & 9 & 38.49 & $0.042^{*}$ \\
\hline \multirow[t]{2}{*}{ Univariate test: } & B. neohumeralis & & B. tryoni & \\
\hline & Deviance & $\operatorname{Pr}(>\mathrm{Dev})$ & Deviance & $\operatorname{Pr}(>\mathrm{Dev})$ \\
\hline latitude & 12.14 & 0.35 & 4.33 & 0.81 \\
\hline status & 48.57 & $0.001^{* * *}$ & 74.50 & $0.001^{* * *}$ \\
\hline latitude:status & 25.73 & 0.128 & 12.76 & 0.128 \\
\hline
\end{tabular}

Table presents Analysis of Deviance for multivariate and univariate analyses of Wolbachia prevalence (status) at different latitudes of two widespread species $B$ neohumeralis and B. tryoni, as per output of the mvabund package. P-value $(\operatorname{Pr}(>\operatorname{Dev}))$ Significance codes: $0{ }^{\prime * * * \prime} 0.001^{\prime * * \prime} 0.01^{\prime * \prime} 0.05^{\prime \prime} .0 .1^{\prime \prime} 1$

over time. In B. neohumeralis and B. tryoni, Wolbachia was not linked with any particular mitochondrial haplotypes. We thus concluded that the detected strains may not necessarily manipulate reproduction of their host species and maternal inheritance may also be unreliable. Therefore, the detection of identical Wolbachia strains at low prevalence in several species of this fruit fly community may be due to a spillover from a yet unknown source species, without the propagation of Wolbachia in any of the analysed host species, and this spillover appears to be restricted to the tropical regions.

\section{Wolbachia strains and pseudogenes}

The previous MLST characterisation in Australian tropical tephritids revealed four complete MLST profiles of supergroup A Wolbachia in seven species [28]. Here, however, 16S rDNA and wsp sequences were isolated from nine and eight species, respectively while complete marker gene sets were not found for all nine species, which is likely to be due to lateral gene transfer of $\mathrm{Wol}$ bachia into the host genome. More specifically, all individuals of $B$. perkinsi produced $16 \mathrm{~S}$ rDNA and wsp sequences (including some wsp sequences with stop codons). In the previous study, no individuals of this species amplified fts $Z$ and produced both pseudogenic and novel ORF sequences for other MLST loci [28]. Another species, B. peninsularis, also had signs of Wolbachia lateral gene transfer, as all individuals only amplified $16 \mathrm{~S}$ rDNA but not wsp in this study while previously $f b p A$ was detected as the only positive MLST locus in all individuals of this species [28]. Similarly, pseudogenised $16 \mathrm{~S}$ rDNA and $f b p A$ sequences were found in one out of eight tested Glossina fly species [15]. We thus conclude that the Wolbachia signals in B. peninsularis and B.perkinsi are due to lateral gene transfer from Wolbachia into these host genomes, as has been reported for other insects [14]. It may also be noted that the Wolbachia pseudogenic sequences were different enough to conclude that they did not derive from the four strains detected in this study.

Two of the previously characterised Wolbachia strains ST-285 and ST-289 [28] were found with their 16S rDNA and wsp sequences in individuals of six fruit fly species. Single infections by both of these strains were detected in B. neohumeralis individuals and single infections by ST-285 in all four infected B. tryoni individuals and one B. frauenfeldi individual. Two additional strains, ST-17 and ST-370, previously characterised by Morrow et al. [28] were detected with their 16S rDNA and wsp sequence variants in another $B$. frauenfeldi individual. It was demonstrated that this double infection is identical to Wolbachia from the fruit fly parasitoid F. arisanus, and this could be a spillover from this parasitoid species. This conclusion can be drawn based on the result of our earlier study in which all individuals of this parasitoid species were infected, including individuals from the Sydney region where fruit fly host populations were uninfected [28]. Of additional interest is that both $F$. arisanus and B. frauenfeldi are not endemic to Australia; $F$. arisanus was introduced to Australia in 1956 and now occurs from northern Queensland to Sydney [36, 5455] while B. frauenfeldi was first detected on Cape York Peninsula in 1974 from where it has spread throughout northern Queensland [55]. This also means that ST-17 and ST-370 were only found in two host species that were originally not native to Australia.

\section{Latitudinal distribution of Wolbachia in Australian tephritids}

After excluding pseudogenes in two species as potential Wolbachia infections, we detected Wolbachia in $29 \%$ of tested Australian tephritid species, and this is lower than previous estimates of $40 \%$ incidence of infections in arthropods, mostly based on a European dataset [1, 48]. 


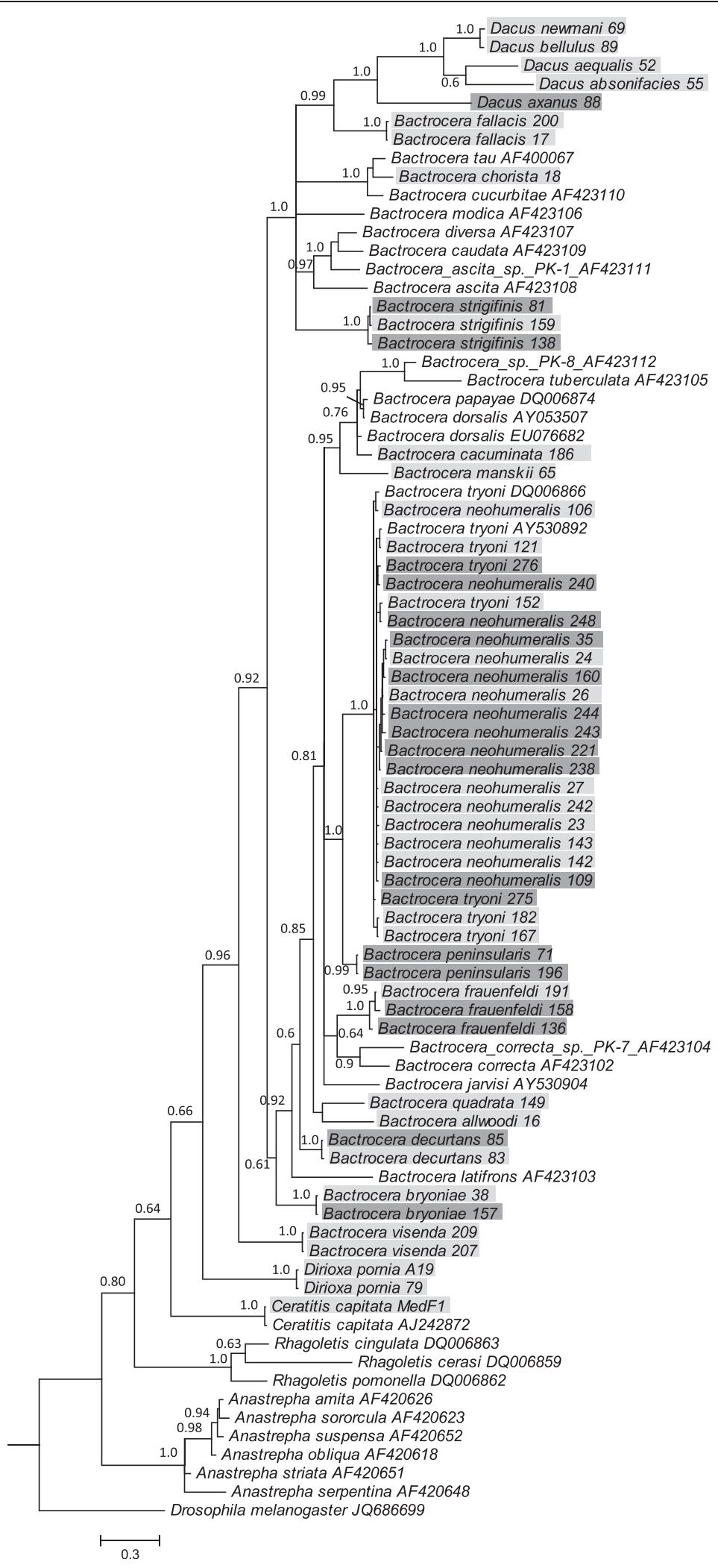

Fig. 3 (See legend on next page.) 
(See figure on previous page.)

Fig. 3 Bayesian inference tree of $\mathrm{CO}$ sequences from tephritid fruit flies. Representatives of the five major tephritid fruit fly genera, Anastrepha, Bactrocera, Ceratitis, Dacus and Rhagoletis as well as Dirioxa are shown. Dark grey shading highlights the individuals from this study harbouring Wolbachia, light grey highlights specimens that were negative for Wolbachia. Sequences from species without shading were retrieved from GenBank (accession numbers shown. Note: name for B. dorsalis has been retained as B. papayae DQ006874 as per GenBank entry, although these species names have been synonymised [40]). Tree was rooted with Drosophila melanogaster, and nodes are labelled with posterior probability values. Scale bar represents the number of nucleotide substitutions per site

Our results varied between climatic regions. Wolbachia incidence was restricted to equatorial and tropical regions north of Gladstone, while Wolbachia was absent from individuals caught in the more southern subtropical, temperate and arid inland regions. Specifically, Wolbachia was found in species whose range is limited to tropical and equatorial Queensland (B. decurtans, B. frauenfeldi, B. strigifinis); in species with a broader climatic range, Wolbachia occurred as far south as Cairns for B. bryoniae, Mackay for B. tryoni and Gladstone for B. neohumeralis, but was absent from individuals in more southerly subtropical and temperate regions. Within all of these species, Wolbachia prevalence was very low (2-17\%), and in the more widely distributed species $B$. neohumeralis and $B$. tryoni, Wolbachia was also restricted to the northern ranges of each species.

\section{Wolbachia in Australian tephritids: transient infections resulting from a spillover?}

Previously, identical Wolbachia strains were found in multiple species of fruit flies that share ecological niches, but are unlikely to hybridise in nature, except possibly for B. tryoni and B. neohumeralis [28]. This study found that only low frequency Wolbachia signals were detected in samples from tropical environments, including for those species that are widespread from tropical to temperate climes. Furthermore, no Wolbachia was detected in any laboratory colonies of these species, including those originating from tropical locations, a result that was supported by 454 pyrosequencing of $16 \mathrm{~S}$ rDNA amplicon libraries from these same individuals [41]. This suggests that there is ample opportunity for the horizontal transfer of Wolbachia, but the low prevalence, and lack of increasing prevalence over time, does not unequivocally show that these infections confer any selective advantage to infected females e.g. through reproductive manipulation such as cytoplasmic incompatibility (CI) nor that they are being reliably inherited. Experimental evidence of a heritable Wolbachia infection is necessary to confirm an established infection. In Drosophila suzukii, a similarly low frequency Wolbachia infection occurs with variable maternal transmission; however no CI or fitness benefits (such as increased fecundity, starvation resistance or desiccation resistance) were observed, and maintenance of Wolbachia in this species has yet to be explained [56]. Here, in the absence of any evidence of vertical inheritance, the finding of two strains (ST-285 and ST-289) in six host species at low prevalence is difficult to explain, and perhaps more likely due to a spillover of Wolbachia from an as yet unknown host species without any proliferation in any of the studied species. This interpretation still lacks direct experimental evidence, but transient infections are frequently observed in Wolbachia microinjection studies that detect Wolbachia in many individuals emerging from microinjected embryos. However, only a few injected embryos result in the establishment of stably inherited matrilines while Wolbachia is lost from most other matrilines [11], or remains undetectable in some matrilines for many generations [57]. In our study, the potential spillover of Wolbachia is restricted to the tropical regions, and is perhaps due to the higher biodiversity, resulting in increased ecological interactions in the tropics. The finding of two strains (ST-17 and ST-370) that are shared between fruit fly and parasitoid individuals supports the interpretation of a potential spillover with the parasitoid being the source in this instance; ST-17 and ST370 were only found in one $B$. frauenfeldi individual while all $F$. arisanus individuals were infected with both strains, including from regions where host fruit flies were uninfected [28]. If, however, the infections are heritable in the studied fruit fly species, then the latitudinal restriction of Wolbachia incidence and prevalence in Australian fruit flies to northern Australia may be due to (1) Wolbachia loss due to environmental conditions of their hosts in the southern range or (2) an ongoing Wolbachia invasion of fruit fly species from north to south, driven by a reproductive phenotype.

An increasing number of studies have demonstrated that Wolbachia responds to the climatic environment of its host insects. For example, precipitation frequency appeared to correlate with the distribution of single and double infections in leaf beetle individuals in Panama, with multiple infections restricted to wetter regions [58]. In another study, multiple infections were found to be more frequent in one tropical habitat when compared with two temperate habitats [20]. Temperature is known to affect Wolbachia titres in a number of insect hosts. For example, the virulent $w$ MelPop strain appears to over-replicate at elevated temperatures [59]. However, high temperatures may also effectively reduce Wolbachia densities in hosts through increased Wolbachiabacteriophage activity [60], and thus result in diminished penetrance of CI or male-killing phenotypes [61, 62]. It is possible that temperature fluctuations and extremes, commonly found in southern regions of Australia, may 
be selecting more strongly against Wolbachia than the more constant temperature conditions in the tropics. Such climatic effects could also be contributing to the distribution of Wolbachia in previously reported analyses such as D. melanogaster [26] and cat fleas [63]. Similarly, climate and latitude were found to determine ranges of other microbial symbioses, for example in marine invertebrates [64], terrestrial insects [65-67] and humans [68].

The second scenario supposes that the Wolbachia in the Australian tephritid species, in particular infections of the widespread B. tryoni and B. neohumeralis, is relatively recently acquired, and a progressive $\mathrm{CI}$ driven sweep may therefore be in its infancy. However this hypothesis is less likely for the following reasons; a CI driven invasion should result in an increase of infection prevalence over time that was not detected in these species. Furthermore, Wolbachia-induced CI is expected to cause a selective sweep of infected mitochondrial haplotypes [69], yet there does not appear to be support for this in the B. tryoni and B. neohumeralis species complex [38] because both species share mitochondrial haplotypes across infected and uninfected individuals. We were not able to directly assess the CI phenotype of Wolbachia in Australian tephritids due to the lack of infected laboratory populations. The CI characterisation of the Wolbachia strains will thus require future field collection efforts, in particular of females, in order to set up infected laboratory colonies for crossing experiments. Testing of field females will also avoid a potential underestimate of Wolbachia infection rates due to our male-biased sampling approach that would not detect male-killing Wolbachia strains. However, prevalence of male-killing Wolbachia may be generally low as found for another fruit fly family, Drosophilidae [70].

\section{Evidence for numtDNA in Bactrocera}

Blacket et al. [71] have previously detected numtDNA amplicons for B. tryoni and B. neohumeralis by using primers for a different section of COI; our different primer set did not yield numtDNA for B. tryoni and B. neohumeralis but revealed potential numtDNA sequences in other species such as B. jarvisi, B. dorsalis, B. murrayi and B. perkinsi. Interestingly, potential transfer of both mitochondrial and Wolbachia genes into the nuclear host genome were detected for B. perkinsi.

\section{Conclusions}

In this study of Australian tephritids we detected several Wolbachia pseudogenes that may be host-genomic footprints of historic infections that have since been lost from host species. Within the species of relevance $(B$. peninsularis and $B$. perkinsi), these pseudogenes were fixed at $100 \%$ while prevalence of Wolbachia infections in other species was overly low $(<20 \%)$. Under the assumption that the detected Wolbachia strains in seven fruit fly species may not be reliably inherited nor cause a reproductive phenotype, these infections could be considered transient and a result of a spillover from a yet unknown source (while we have identified a potential Wolbachia spillover from $F$. arisanus to B. frauenfeldi). In our study system, this spillover appears to be restricted to northern Australia and has not resulted in further proliferation within any of the tested host lineages, and this warrants future investigation. The fact that we have not found a single case of a high prevalence infected Australian tephritid fruit fly species in 24 tested species raises the question whether these taxa exhibit traits that reduce Wolbachia infectivity while two species had Wolbachia infections in their evolutionary past. Other related pest taxa such as olive fly Bactrocera oleae appear uninfected in natural populations while laboratory populations were successfully infected [72]; a high incidence of wsp genes was found in Thai Bactrocera species $[73,74]$ while prevalence of $w s p$ in $B$. dorsalis was found to be extremely low [21], although these strains were not further characterised. Perhaps some of these Bactrocera species also contain Wolbachia signals that are either pseudogenes and/or transient somatic infections, and similar questions may be valid for Wolbachia signals found in other host species. Our study highlights that tropical insect communities have signs of lateral gene transfer and may be exposed to increased horizontal Wolbachia transfer. It also emphasises that global estimates for Wolbachia infection frequencies should be interpreted with caution, and account for lateral gene transfer, variable prevalence within species and transient spillover effects. This may be of particular relevance if Wolbachia surveys are based on a single or a few marker genes for detection of infections in a host species, and without a broader sampling effort from the host's distribution (also see [75]).

\section{Availability of supporting data}

Data sets supporting the results of this article are included within the article and its additional files. DNA sequences generated in this study were submitted to GenBank: accession numbers KC581371-KC581411, KC693011, KC775785-KC775792 (COI) and KC668319KC668321, KC668323-KC668332, KC693012 (wsp) and KC775793-KC775795 (16S rDNA). New wsp alleles have also been deposited in the Wolbachia wsp database (http://pubmlst.org/wolbachia/wsp/).

\section{Additional files}

Additional file 1: Collection data for field-caught fruit flies from this study. Information for collection location, climate group and year, specimen 
specific ID number, sex, result for wsp and 165 rDNA screening and GenBank accession number of COl partial gene are included. (CSV $61 \mathrm{~kb}$ )

Additional file 2: Laboratory strains screened for Wolbachia using wsp and 16S rDNA. (PDF $104 \mathrm{~kb}$ )

Additional file 3: PCR conditions and primer sequences used in this research. (PDF $103 \mathrm{~kb}$ )

Additional file 4: Relationship between prevalence of Wolbachia infection in twelve tephritid species and the midpoint of their latitudinal distribution, based on Hancock et al. [32] and Royer and Hancock [33] $\left(\mathrm{R}^{2}=0.86, \mathrm{~F}_{1,10}=71.43 ; p<0.001\right)$. At least ten individuals were tested for uninfected species (B. cacuminata, $B$. chorista, B. jarvisi, D. aequalis, D. pornia). (PDF $126 \mathrm{~kb}$ )

Additional file 5: Comparison of Wolbachia infected individuals sampled from equatorial and tropical regions for (A) B. bryoniae, (B) B. frauenfeldi, (C) B. neohumeralis, (D), B. strigifinis and (E) B. tryoni over five collection years. Fisher's exact test shows overall no significant (ns) temporal effect at $a=0.05$, except for $B$. tryoni, but this did not represent an overall increase or decrease in prevalence. (PDF $100 \mathrm{~kb}$ )

Additional file 6: Distance matrix of $\mathrm{COI}$ sequences covering 551 bp positions of $B$. tryoni and $B$. neohumeralis individuals, grouped by Wolbachia-infected (grey shading) and uninfected (no shading). Below the diagonal are the pairwise number of differences and above the diagonal are $\mathrm{p}$-distance values. P-distances given for within- and between- group means are listed below the main table. (PDF $103 \mathrm{~kb}$ )

\section{Competing interests}

The authors declare that they have no competing interests.

\section{Authors' contributions}

The project was conceived by MR and JLM; the research was designed by $J L M$ and MR with input from MF and DCAS; JLM performed the research and analysed the data; MR, MF, JER and DCAS supplied materials and specimens; $J L M$ and MR wrote the paper with input by MF, JER and DCAS. All authors read and approved the final manuscript.

\section{Acknowledgements}

We thank Sally Cowan (DA - Biosecurity) and lan Lacey (Agriculture and Food, Western Australia) for supplying fruit fly specimens as well as the CSIRO Double Helix Club for earlier fruit fly collections. We acknowledge Goran Lopaticki for maintenance of laboratory colonies, Jeff Powell, Andrew Gherlenda, John Sved and David Warton for statistical advice. JLM was supported by an Australian Postgraduate and a FG Swain Award, and MR by research funds from HIE.

\section{Author details}

'Hawkesbury Institute for the Environment, Western Sydney University, Locked Bag 1797, Penrith, NSW 2751, Australia. ${ }^{2}$ Evolution and Ecology Research Centre, School of Biological, Earth and Environmental Sciences, University of New South Wales, Sydney, NSW 2052, Australia. ${ }^{3}$ Queensland Department of Agriculture and Fisheries, EcoSciences Precinct, 41 Boggo Road, Dutton Park, QLD 4102, Australia.

Received: 28 May 2015 Accepted: 3 September 2015

Published online: 18 September 2015

\section{References}

1. Zug R, Hammerstein P. Still a host of hosts for Wolbachia: Analysis of recent data suggests that $40 \%$ of terrestrial arthropod species are infected. PLoS One. 2012;7(6):e38544.

2. Weinert LA, Araujo-Jnr EV, Ahmed MZ, Welch JJ. The incidence of bacterial endosymbionts in terrestrial arthropods. Proc R Soc Lond B Biol Sci. 2015;282(1807):20150249

3. Werren JH, Baldo L, Clark ME. Wolbachia: master manipulators of invertebrate biology. Nat Rev Microbiol. 2008;6(10):741-51.

4. Schuler H, Bertheau C, Egan SP, Feder JL, Riegler M, Schlick-Steiner BC, et al. Evidence for a recent horizontal transmission and spatial spread of Wolbachia from endemic Rhagoletis cerasi (Diptera: Tephritidae) to invasive Rhagoletis cingulata in Europe. Mol Ecol. 2013;22(15):4101-11.
5. Zug R, Koehncke A, Hammerstein P. Epidemiology in evolutionary time: The case of Wolbachia horizontal transmission between arthropod host species. J Evol Biol. 2012;25(11):2149-60.

6. Raychoudhury R, Baldo L, Oliveira DC, Werren JH. Modes of acquisition of Wolbachia: horizontal transfer, hybrid introgression, and codivergence in the Nasonia species complex. Evolution. 2009;63(1):165-83.

7. Rousset F, Solignac M. Evolution of single and double Wolbachia symbioses during speciation in the Drosophila simulans complex. Proc Natl Acad Sci U S A. 1995;92(14):6389-93.

8. Rigaud T, Juchault P. Success and failure of horizontal transfers of feminizing Wolbachia endosymbionts in woodlice. J Evol Biol. 1995;8(2):249-55.

9. Werren JH, Zhang W, Guo LR. Evolution and phylogeny of Wolbachia: reproductive parasites of arthropods. Proc $\mathrm{R}$ Soc Lond B Biol Sci. 1995:261:55-63.

10. Combes C. Parasitism: the ecology and evolution of intimate interactions. University of Chicago Press: Chicago, USA; 2001.

11. Riegler M, Charlat S, Stauffer C, Mercot H. Wolbachia transfer from Rhagoletis cerasi to Drosophila simulans: investigating the outcomes of host-symbiont coevolution. Appl Environ Microbiol. 2004;70(1):273-9.

12. Otterstatter MC, Thomson JD. Does pathogen spillover from commercially reared bumble bees threaten wild pollinators? PLoS One. 2008;3(7):e2771.

13. Arthofer W, Riegler M, Avtzis DN, Stauffer C. Evidence for low-titre infections in insect symbiosis: Wolbachia in the bark beetle Pityogenes chalcographus (Coleoptera, Scolytinae). Environ Microbiol. 2009;11(8):1923-33.

14. Dunning Hotopp JC, Clark ME, Oliveira DC, Foster JM, Fischer P, Munoz Torres MC, et al. Widespread lateral gene transfer from intracellular bacteria to multicellular eukaryotes. Science. 2007;317(5845):1753-6.

15. Doudoumis V, Tsiamis G, Wamwiri F, Brelsfoard C, Alam U, Aksoy E, et al. Detection and characterization of Wolbachia infections in laboratory and natural populations of different species of tsetse flies (genus Glossina). BMC Microbiol. 2012;12 Suppl 1:S3.

16. Novotny $V$, Drozd $P$, Miller SE, Kulfan $M$, Janda $M$, Basset $Y$, et al. Why are there so many species of herbivorous insects in tropical rainforests? Science. 2006;313(5790):1115-8.

17. Lewinsohn TM, Roslin T. Four ways towards tropical herbivore megadiversity. Ecol Lett. 2008;11(4):398-416.

18. Werren JH, Windsor DM, Guo LR. Distribution of Wolbachia among neotropical arthropods. Proc R Soc Lond B Biol Sci. 1995;262(1364):197-204.

19. West SA, Cook JM, Werren JH, Godfray HC. Wolbachia in two insect hostparasitoid communities. Mol Ecol. 1998;7(11):1457-65.

20. Werren $\mathrm{JH}$, Windsor DM. Wolbachia infection frequencies in insects: evidence of a global equilibrium? Proc R Soc Lond B Biol Sci. 2000;267(1450):1277-85

21. Ahmed MZ, Greyvenstein OFC, Erasmus C, Welch JJ, Greeff JM. Consistently high incidence of Wolbachia in global fig wasp communities. Ecol Entomol. 2013;38(2):147-54.

22. Sun X, Cui L, Li Z. Diversity and phylogeny of Wolbachia infecting Bactrocera dorsalis (Diptera: Tephritidae) populations from China. Environ Entomol. 2007;36(5):1283-9.

23. Riegler M, Stauffer C. Wolbachia infections and superinfections in cytoplasmically incompatible populations of the European cherry fruit fly Rhagoletis cerasi (Diptera, Tephritidae). Mol Ecol. 2002;11(11):2425-34.

24. Miller WJ, Ehrman L, Schneider D. Infectious speciation revisited: impact of symbiont-depletion on female fitness and mating behavior of Drosophila paulistorum. PLoS Pathog. 2010;6(12):e1001214.

25. Hoffmann AA, Clancy DJ, Merton E. Cytoplasmic incompatibility in Australian populations of Drosophila melanogaster. Genetics. 1994;136(3):993-9.

26. Hoffmann AA, Hercus M, Dagher H. Population dynamics of the Wolbachia infection causing cytoplasmic incompatibility in Drosophila melanogaster. Genetics. 1998;148(1):221-32.

27. Kriesner P, Hoffmann AA, Lee SF, Turelli M, Weeks AR. Rapid sequential spread of two Wolbachia variants in Drosophila simulans. PLOS Pathog. 2013;9(9):e1003607.

28. Morrow JL, Frommer M, Shearman DCA, Riegler M. Tropical tephritid fruit fly community with high incidence of shared Wolbachia strains as platform for horizontal transmission of symbionts. Environ Microbiol. 2014;16(12):3622-37.

29. White IM, Elson-Harris MM. Fruit flies of economic significance: their identification and bionomics. Oxon, UK: CAB International; 1992.

30. Drew RAl. The tropical fruit flies (Diptera: Tephritidae: Dacinae) of the Australasian and Oceanian regions. Memoirs Queensland Museum. 1989;26:1-521.

31. Hancock DL. A revised checklist of Australian fruit flies (Diptera: Tephritidae). Aust Entomol. 2013;40(4):219-36. 
32. Hancock DL, Hamacek EL, Lloyd AC, Elson-Harris MM. The distribution and host plants of fruit flies (Diptera: Tephritidae) in Australia. Department of Primary Industries, Queensland: Brisbane, Queensland; 2000

33. Royer JE, Hancock DL. New distribution and lure records of Dacinae (Diptera: Tephritidae) from Queensland, Australia, and description of a new species of Dacus Fabricius. Aust J Entomol. 2012;51(4):239-47.

34. Meats A. The bioclimatic potential of the Queensland fruit fly, Dacus tryoni, in Australia. Proc Ecol Soc Aust. 1981;11:151-61.

35. Vijaysegaran $\mathrm{S}$, Walter GH, Drew RAl. Mouthpart structure, feeding mechanisms, and natural food sources of adult Bactrocera (Diptera: Tephritidae). Ann Ent Soc Am. 1997;90(2):184-201

36. Carmichael AE, Wharton RA, Clarke AR. Opiine parasitoids (Hymenoptera: Braconidae) of tropical fruit flies (Diptera: Tephritidae) of the Australian and South Pacific region. Bull Entomol Res. 2005;95(6):545-69.

37. Osborne R, Meats A, Frommer M, Sved JA, Drew RAl, Robson MK. Australian distribution of 17 species of fruit flies (Diptera: Tephritidae) caught in cue lure traps in February 1994. Aust J Entomol. 1997:36(1):45-50.

38. Morrow J, Scott L, Congdon B, Yeates D, Frommer M, Sved J. Close genetic similarity between two sympatric species of tephritid fruit fly reproductively isolated by mating time. Evolution. 2000;54(3):899-910.

39. Cameron EC, Sved JA, Gilchrist AS. Pest fruit fly (Diptera: Tephritidae) in northwestern Australia: One species or two? Bull Entomol Res. 2010;100(2):197-206.

40. Schutze MK, Aketarawong N, Amornsak W, Armstrong KF, Augustinos AA, Bar $\mathrm{N}$, et al. Synonymization of key pest species within the Bactrocera dorsalis species complex (Diptera: Tephritidae): taxonomic changes based on a review of 20 years of integrative morphological, molecular, cytogenetic, behavioural and chemoecological data. Syst Entomol. 2015;40:456-71.

41. Morrow J, Frommer M, Shearman D, Riegler M. The microbiome of fieldcaught and laboratory-adapted Australian tephritid fruit fly species with different host plant use and specialisation. Microb Ecol 2015;70(2):498-508.

42. Min KT, Benzer S. Wolbachia, normally a symbiont of Drosophila, can be virulent, causing degeneration and early death. Proc Natl Acad Sci U S A. 1997;94(20):10792-6.

43. Duplouy A, Vermenot C, Davies N, Roderick G, Hurst GDD, Charlat S. Assessing risks of Wolbachia DNA cross-specimen contamination following mass collection and ethanol storage. Mol Ecol Res. 2009;9(1):46-50.

44. Braig HR, Zhou W, Dobson SL, O'Neill SL. Cloning and characterization of a gene encoding the major surface protein of the bacterial endosymbiont Wolbachia pipientis. J Bacteriol. 1998;180(9):2373-8.

45. Jeyaprakash A, Hoy MA. Long PCR improves Wolbachia DNA amplification: wsp sequences found in $76 \%$ of 63 arthropod species. Insect Mol Biol. 2000;9(4):393-405

46. Simon C, Frati F, Beckenbach A, Crespi B, Liu H, Flook P. Evolution, weighting, and phylogenetic utility of mitochondrial gene sequences and a compilation of conserved polymerase chain reaction primers. Ann Ent Soc Am. 1994;87(6):651-701.

47. Arthofer W, Riegler M, Schneider D, Krammer M, Miller WJ, Stauffer C. Hidden Wolbachia diversity in field populations of the European cherry fruit fly, Rhagoletis cerasi (Diptera, Tephritidae). Mol Ecol. 2009;18(18):3816-30.

48. Duron O, Bouchon D, Boutin S, Bellamy L, Zhou L, Engelstädter J, et al. The diversity of reproductive parasites among arthropods: Wolbachia do not walk alone. BMC Biol. 2008;6:27.

49. Wang Y, Naumann U, Wright ST, Warton DI. mvabund- an R package for modelbased analysis of multivariate abundance data. Meth Ecol Evol. 2012;3(3):471-4

50. R Core Team. R: A Language and Environment for Statistical Computing. Vienna, Austria: R Foundation for Statistical Computing; 2012

51. Tamura K, Peterson D, Peterson N, Stecher G, Nei M, Kumar S. MEGA5: molecular evolutionary genetics analysis using maximum likelihood, evolutionary distance, and maximum parsimony methods. Mol Biol Evol. 2011;28(10):2731-9.

52. Ronquist F, Teslenko M, van der Mark P, Ayres DL, Darling A, Höhna S, et al. MrBayes 3.2: efficient Bayesian phylogenetic inference and model choice across a large model space. Syst Biol. 2012;61(3):539-42.

53. Baldo L, Dunning Hotopp JC, Jolley KA, Bordenstein SR, Biber SA, Choudhury $\mathrm{RR}$, et al. Multilocus sequence typing system for the endosymbiont Wolbachia pipientis. Appl Environ Microbiol. 2006;72(11):7098-110.

54. Snowball GJ, Wilson F, Campbell TG, Lukins RG. The utilization of parasites of oriental fruit fly (Dacus dorsalis) against Queensland fruit fly (Strumeta tryoni). Crop Pasture Sci. 1962;13(3):443-7.

55. Royer JE, Wright CL, Hancock DL. Bactrocera frauenfeldi (Diptera: Tephritidae), an invasive fruit fly in Australia that may have reached the extent of its spread due to environmental variables. Austral Entomol 2015; doi:10.1111/aen.12155

56. Hamm CA, Begun DJ, Vo A, Smith CC, Saelao P, Shaver AO, et al. Wolbachia do not live by reproductive manipulation alone: infection polymorphism in Drosophila suzukii and D. subpulchrella.

57. Schneider DI, Riegler M, Arthofer W, Merçot H, Stauffer C, Miller WJ. Uncovering Wolbachia diversity upon artificial host transfer. PLoS One 2013;8(12):e82402.

58. Keller GP, Windsor DM, Saucedo JM, Werren JH. Reproductive effects and geographical distributions of two Wolbachia strains infecting the Neotropical beetle, Chelymorpha alternans Boh. (Chrysomelidae, Cassidinae). Mol Ecol. 2004;13(8):2405-20.

59. Reynolds KT, Thomson $\sqcup$, Hoffmann AA. The effects of host age, host nuclear background and temperature on phenotypic effects of the virulent Wolbachia strain popcorn in Drosophila melanogaster. Genetics. 2003;164(3):1027-34

60. Bordenstein SR, Bordenstein SR. Temperature affects the tripartite interactions between bacteriophage WO, Wolbachia, and cytoplasmic incompatibility. PLoS One. 2011;6(12):e29106

61. Hurst GDD, Johnson AP, von der Schulenburg JHG, Fuyama Y. Male-killing Wolbachia in Drosophila: a temperature-sensitive trait with a threshold bacterial density. Genetics. 2000;156(2):699-709.

62. Breeuwer JAJ, Werren JH. Cytoplasmic incompatibility and bacterial density in Nasonia vitripennis. Genetics. 1993;135(2):565-74

63. Tay ST. Wolbachia endosymbionts, Rickettsia felis and Bartonella species, in Ctenocephalides felis fleas in a tropical region. J Vect Ecol. 2013;38(1):200-2.

64. Sanders JG, Palumbi SR. Populations of Symbiodinium muscatinei show strong biogeographic structuring in the intertidal anemone Anthopleura elegantissima. Biol Bull. 2011;220(3):199-208.

65. Dunbar HE, Wilson ACC, Ferguson NR, Moran NA. Aphid thermal tolerance is governed by a point mutation in bacterial symbionts. PLoS Biol. 2007:5(5):e96.

66. Morag N, Klement E, Saroya Y, Lensky I, Gottlieb Y. Prevalence of the symbiont Cardinium in Culicoides (Diptera: Ceratopogonidae) vector species is associated with land surface temperature. FASEB J. 2012;26(10):4025-34.

67. Mueller UG, Mikheyev AS, Hong E, Sen R, Warren DL, Solomon SE, et al. Evolution of cold-tolerant fungal symbionts permits winter fungiculture by leafcutter ants at the northern frontier of a tropical ant-fungus symbiosis. Proc Natl Acad Sci U S A. 2011;108(10):4053-6.

68. Guernier V, Hochberg ME, Guégan J-F. Ecology drives the worldwide distribution of human diseases. PLoS Biol. 2004;2(6):e141.

69. Hurst GDD, Jiggins FM. Problems with mitochondrial DNA as a marker in population, phylogeographic and phylogenetic studies: the effects of inherited symbionts. Proc R Soc Lond B Biol Sci. 2005;272(1572):1525-34

70. Hurst G, Jiggins FM. Male-killing bacteria in insects: mechanisms, incidence and implications. Emerg Infect Dis. 2000;6(4):329-36.

71. Blacket MJ, Semeraro L, Malipatil MB. Barcoding Queensland fruit flies (Bactrocera tryoni): impediments and improvements. Mol Ecol Res. 2012;12(3):428-36.

72. Apostolaki A, Livadaras I, Saridaki A, Chrysargyris A, Savakis C, Bourtzis K Transinfection of the olive fruit fly Bactrocera oleae with Wolbachia: towards a symbiont-based population control strategy. J App Ent. 2011;135(7):546-53.

73. Jamnongluk W, Kittayapong P, Baimai V, O'Neill SL. Wolbachia infections of tephritid fruit flies: molecular evidence for five distinct strains in a single host species. Curr Microbiol. 2002;45(4):255-60.

74. Kittayapong P, Milne JR, Tigvattananont S, Baimai V. Distribution of the reproduction-modifying bacteria, Wolbachia, in natural populations of tephritid fruit flies in Thailand. ScienceAsia. 2000;26:93-103.

75. Nguyen DT, Spooner-Hart RN, Riegler M. Polyploidy versus endosymbionts in obligately thelytokous thrips. BMC Evol Biol. 2015;15:23. 